\title{
Adaptive transgenerational effects remain significant
}

\author{
Yuan-Ye Zhang ${ }^{1}$, Junjie Yin ${ }^{1}$, Ming Zhou ${ }^{1}$, Zeru Lin $^{1}$, and Qingshun Q. Li $^{1}$ \\ ${ }^{1}$ Key Laboratory of the Ministry of Education for Coastal and Wetland Ecosystems, \\ Xiamen University
}

July 16, 2020

\begin{abstract}
The comment by Sánchez-Tójar et al. (2020, Ecol Lett) questioned the methodology, transparency, and conclusion of our study (Yin et al. 2019, Ecol Lett, 22, 1976). The comment has overlooked important evolutionary assumptions in their reanalysis, and the issues raised were in fact dealt with through the peer-review process. Far from being biased, the key conclusion of our meta-analysis still stands; transgenerational effects are largely adaptive.
\end{abstract}

Three concerns have been raised by Sánchez-Tójar et al . (2020) about our meta-analysis of transgenerational effects (Yin et al . 2019): (1) the analysis did not fully account for the non-independence among data, (2) the study lacked transparency and the peer review was ineffective, and (3) the literature search had limited coverage. These concerns are addressed below.

First, Sánchez-Tójar et al . raised concerns regarding data non-independence, and in their reanalysis, they attempted to account for phylogenetic non-independence by assuming a Brownian motion model of trait evolution. The revised estimates for effect sizes were largely consistent with our own, and all remained significant except when accounting for phylogenetic non-independence (Fig. 1). However, applying this analysis to our data is problematic for three critical reasons. First, the phylogenetic non-independence assumes that traits diverge over time from the ancestral trait of a common ancestor (Felsenstein 1985). This assumption is violated by our data, as most traits are non-homologous (Fig. S1). Second, the Brownian motion model assumes a constant rate of evolution, but this assumption is often unrealistic for traits under selection (Felsenstein 1985). Third, to analyze the data with plants and animals, the Brownian motion model causes great loss of statistic power due to the high variance-covariance estimated (Fig. S2 and S3). If we use other models of trait evolution, such as assuming an accelerating rate of evolution (Blomberg et al . 2003) or assuming incomplete phylogenetic dependence of trait evolution (Pagel 1997), significant results are retained (Fig. 1). Importantly, whether the results are significant or not depends on different assumptions of evolutionary models rather than the data, and notwithstanding the effects of different assumed evolutionary models, the effect size remains consistent across models (Fig. 1). Thus, our main finding still stands that transgenerational effects do indeed seem to be adaptive.

Concerning the transparency of our study and suggestions that the peer-review was ineffective, both the data and R scripts of this study have been provided since the first submission. Given this level of transparency, the reviewers have already commented on most issues raised by Sánchez-Tójar et al ., and we have responded accordingly. These issues include searching in subject areas, dealing with missing keywords, combining data from full-factorial and non-full-factorial experimental designs, and further issues in the supplementary materials. The reviewers also provided constructive suggestions for dealing with difficult issues such as how to decide if a trait is negatively related to fitness. To give even more transparency, a point-by-point reply to reviewers from our initial study submission is provided as supplementary material.

Finally, we agree with Sánchez-Tójar et al . that studies may have been left out of our original meta-analysis. 
Indeed, transgenerational effects are found across the tree of life, indicating that they either have a deep evolutionary origin or have arisen many times (Salinaet al . 2013; Uller et al . 2013). An essential aim of our meta-analysis was to reveal the specific scenarios that give rise to transgenerational effects. Thus, we fully agree that meta-analyses incorporating more data will further our understanding of when and where transgenerational effects are adaptive.

We thank Sánchez-Tójar et al. for their comment, and we hope these discussions will promote open science practice and inspire future comprehensive meta-analyses of transgenerational effects.

\section{ACKNOWLEDGEMENTS}

We thank Da-Yong Zhang, Wayne Dawson, Yanjie Liu, Nianxun Xi, Oliver Bossdorf, and Wenjing Hong for their comments on the manuscript. We also thank the reviewers for the constructive suggestions in the revision. This study is supported by the National Natural Science Foundation of China (31600291) and the Fundamental Research Funds for the Central Universities in China (20720170074).

\section{REFERENCES}

Blomberg, S.P., Garland, T. \& Ives, A.R. (2003). Testing for phylogenetic signal in comparative data: Behavioral traits are more labile. Evolution , 57, 717-745.

Felsenstein, J. (1985). Phylogenies and the comparative method.Am. Nat. , 125, 1-15.

Pagel, M. (1997). Inferring evolutionary processes from phylogenies.Zool. Scr. , 26, 331-348.

Salinas, S., Brown, S.C., Mangel, M. \& Munch, S.B. (2013). Non-genetic inheritance and changing environments. Non-Genet. Inherit. , 1, 38-50.

Sánchez-Tójar, A., Lagisz, M., Moran N.P., Nakagawa, S., Noble, D.W.A \& Reinhold, K. (2020). The jury is still out regarding the generality of adaptive "transgenerational" effects. Ecol. Lett.

Uller, T., Nakagawa, S. \& English, S. (2013). Weak evidence for anticipatory parental effects in plants and animals. J. Evol. Biol. , 26, 2161-2170.

Yin, J., Zhou, M., Lin, Z., Li, Q.Q. \& Zhang, Y.-Y. (2019). Transgenerational effects benefit offspring across diverse environments: a meta-analysis in plants and animals. Ecol. Lett. , 22, 1976-1986.

\section{Figures Legends}

Figure 1 Results of meta-analysis models with different structures of random effects and under different assumptions of evolution. Model 1 uses the raw data in Yin et al . (2019), and all alternative models use the data proved by Sánchez-Tójar et al ., which includes 1059 of the original 1170 effect sizes.Pagel's $0<\lambda<1$ assumes that the phylogenetic dependence of trait evolution is incomplete, and $\lambda$ represents the degree of phylogenetic dependence (Pagel 1997). Blomberg's $0<\mathrm{g}<1$ assumes that the rates of evolution accelerate through time (Blomberg et al. 2003). 


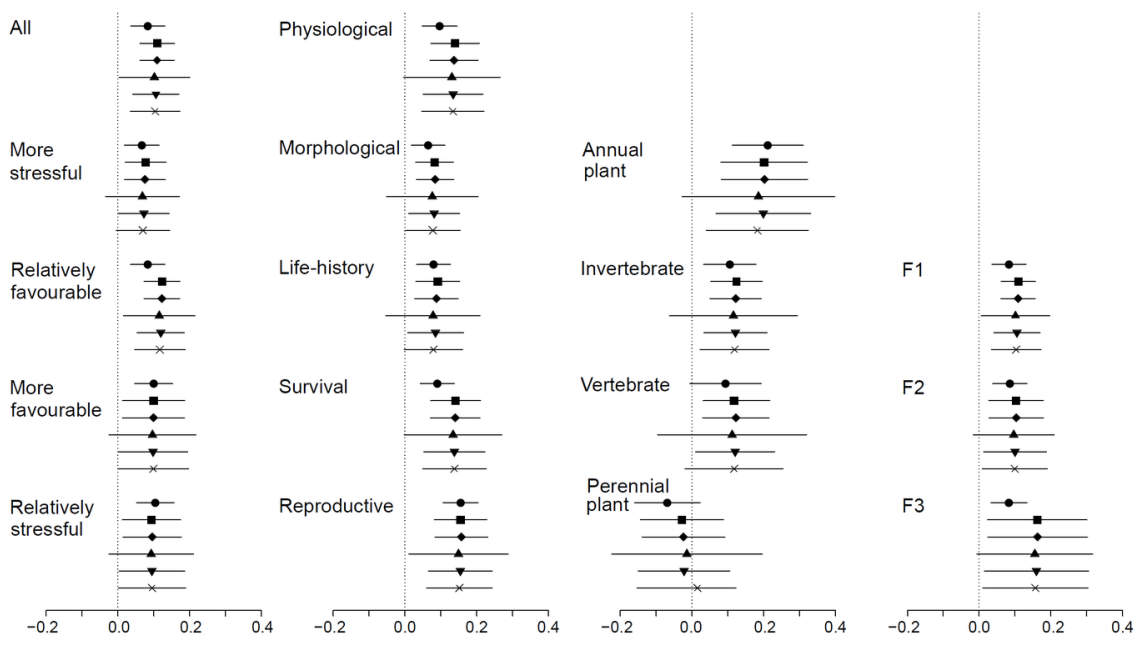

Analysis with the raw data uploaded by Yin et al.

$\rightarrow$ 1. Yin et al. random $=\sim 1 \mid$ species/study

Analysis with the proofed data uploaded by Sánchez-Tójar et al.

$\rightarrow$ 2. Sánchez-Tójar et al. non-conservative approach: random=list( $\sim 1 \mid$ obsID, $\sim 1$ |study, $\sim 1$ |species)

$\rightarrow \quad 3$. Sánchez-Tójar et al. accounting for shared control

ـ 4. Sánchez-Tójar et al. accounting for phylogenetic non-independence (Brownian motion model)

$\rightarrow \quad 5$. accounting for phylogenetic non-independence (Pagel's $\lambda, \lambda=0.5$ )

$\rightarrow 6$. accounting for phylogenetic non-independence (Blomberg's $g, g=0.2$ )

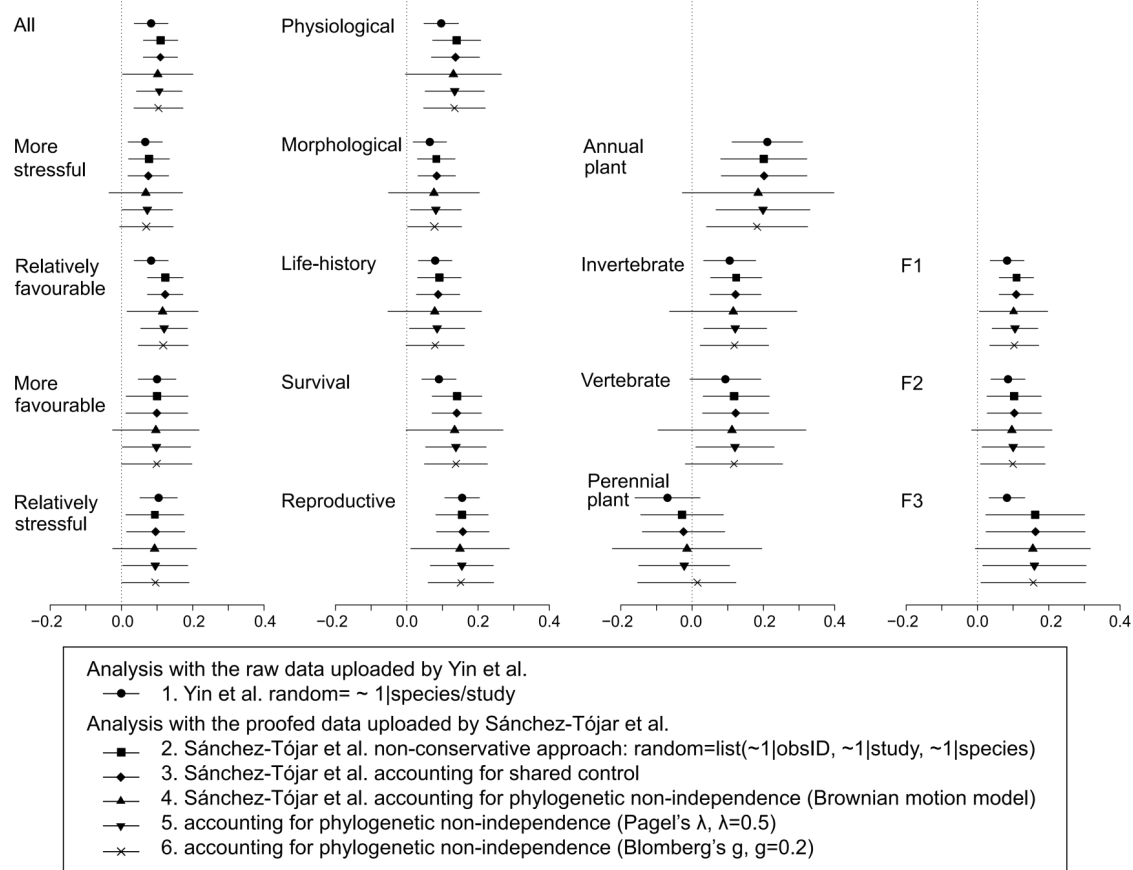

